\title{
Electromagnetic enhancement of ordered silver nanorod arrays evaluated by discrete dipole approximation
}

\author{
Guoke Wei ${ }^{1,2}$, Jinliang Wang ${ }^{1}$ and Yu Chen ${ }^{* 2}$
}

\section{Full Research Paper}

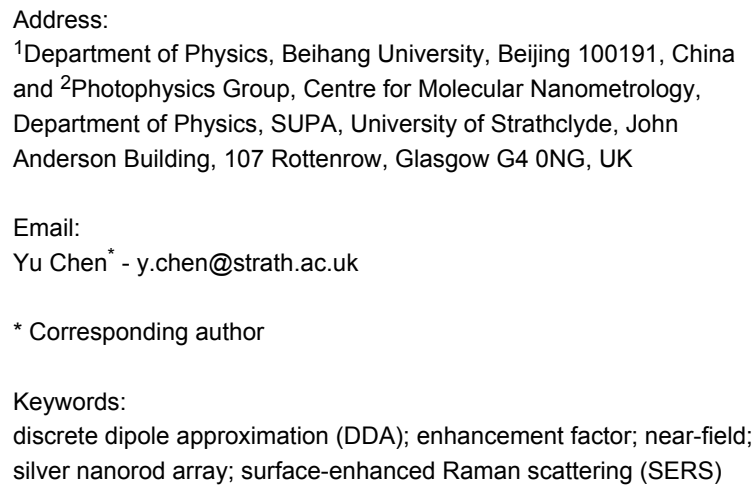

1Department of Physics, Beihang University, Beijing 100191, China and ${ }^{2}$ Photophysics Group, Centre for Molecular Nanometrology, Department of Physics, SUPA, University of Strathclyde, John Anderson Building, 107 Rottenrow, Glasgow G4 ONG, UK

Email:

Yu Chen* - y.chen@strath.ac.uk

* Corresponding author

Keywords:

discrete dipole approximation (DDA); enhancement factor; near-field; silver nanorod array; surface-enhanced Raman scattering (SERS)

Beilstein J. Nanotechnol. 2015, 6, 686-696. doi:10.3762/bjnano.6.69

Received: 15 October 2014

Accepted: 11 February 2015

Published: 09 March 2015

Associate Editor: S. R. Cohen

(c) 2015 Wei et al; licensee Beilstein-Institut. License and terms: see end of document.

\begin{abstract}
The enhancement factor (EF) of surface-enhanced Raman scattering (SERS) from two-dimensional (2D) hexagonal silver nanorod (AgNR) arrays were investigated in terms of electromagnetic (EM) mechanism by using the discrete dipole approximation (DDA) method. The dependence of EF on several parameters, i.e., structure, length, excitation wavelength, incident angle and polarization, and gap size has been investigated. "Hotspots" were found distributed in the gaps between adjacent nanorods. Simulations of AgNR arrays of different lengths revealed that increasing the rod length from 374 to $937 \mathrm{~nm}$ (aspect ratio from 2.0 to 5.0) generated more "hotspots" but not necessarily increased EF under both 514 and $532 \mathrm{~nm}$ excitation. A narrow lateral gap (in the incident plane) was found to result in strong EF, while the dependence of EF on the diagonal gap (out of the incident plane) showed an oscillating behavior. The EF of the array was highly dependent on the angle and polarization of the incident light. The structure of AgNR and the excitation wavelength were also found to affect the EF. The EF of random arrays was stronger than that of an ordered one with the same average gap of $21 \mathrm{~nm}$, which could be explained by the exponential dependence of EF on the lateral gap size. Our results also suggested that absorption rather than extinction or scattering could be a good indicator of EM enhancement. It is expected that the understanding of the dependence of local field enhancement on the structure of the nanoarrays and incident excitations will shine light on the optimal design of efficient SERS substrates and improved performance.
\end{abstract}

\section{Introduction}

Surface-enhanced Raman scattering (SERS) has attracted substantial interest over the past decades due to its potential applications in biological sensing and chemical analysis with molecular specificity and ultrahigh sensitivity, which can be even down to the level of single molecules $[1,2]$. In addition, SERS can be a label-free spectroscopic tool with capabilities in 
real-time and multi-component analysis. Previous studies showed that Raman signals from molecules adsorbed on nanostructured metal surfaces, especially noble metals (e.g., Ag, $\mathrm{Au}$ ), could be amplified by a factor of about $10^{6}$ or even higher [3]. Although the underlying mechanism is still unclear, electromagnetic (EM) enhancement arising from the electric field in the vicinity of noble metal structure is considered as the dominant mechanism for such a dramatic Raman enhancement in most cases [4]. Both theoretical and experimental studies have revealed that the "hotspot", which is the concentration of strong EM fields on nanometre-scale regions with high curvatures or gaps/junctions between closely packed nanoparticles, plays a significant role in SERS enhancements [5,6]. As recently demonstrated by Fang et al., a very small number of molecules residing at the hotspots can dominate the overall SERS signals [7]. Significantly, a single hotspot as small as $15 \mathrm{~nm}$ has been directly measured by single molecule imaging with accuracy down to $1.2 \mathrm{~nm}$ [8].

Tremendous efforts have been devoted to create efficient SERS substrates in recent years [9-11]. Among them, aligned Ag nanorod (AgNR) arrays fabricated by oblique angle deposition (OAD) were shown to be promising SERS substrates with enhancement factors of approximately $10^{8}$ [12-15]. However, the uniformity and reproducibility of SERS substrates remains a major challenge for the applications of SERS. Recently, it has been demonstrated that highly ordered $\mathrm{Ag}$ and $\mathrm{Cu}$ nanorod arrays can be fabricated by a guided OAD method, which may circumvent the problems of gap-size and diameter control, leading to the reproducible fabrication of highly SERS-active substrates [16].

The SERS enhancement not only depends on the intrinsic properties and the dielectric environment of the metal nanoparticles, but also on their shape, size and spatial arrangement. The incident wavelength, angle and polarization were also proven to greatly affect the performance of an SERS substrate. Previously, Chaney et al. observed that the SERS intensity was dramatically enhanced when the nanorod length increased from 190 to $508 \mathrm{~nm}$ in the random AgNR arrays prepared by OAD method. The high aspect ratio and the lateral overlap between adjacent nanorods were considered as the main factors responsible for this phenomenon [12]. Later studies demonstrated that there was an optimal length for the SERS enhancement in the OAD AgNR array [13]. A zig-zag AgNR structure that could generate hotspots at sharp corners also showed potential in enhancing the SERS performance [17]. So far, the understanding of the SERS mechanism in OAD AgNR arrays is still limited. In addition to EM mechanism, surface effect and anisotropic absorbance of molecules were proposed to interpret the SERS enhancement from the AgNR array substrate [18].
Limited systematic studies on OAD AgNR array structures and different measurement conditions used in experimental studies hindered the direct comparison.

Here, we took a systematic approach to investigate the SERS enhancements of the two-dimensional (2D) AgNR arrays from the perspective of EM enhancement mechanism by using the discrete dipole approximation (DDA) method [19]. We expect that the understanding of the dependence of local field enhancement on the structure of the nanoarrays and incident excitations will shine light on the optimal design of efficient SERS substrates and facilitate their applications in biomedical sensing and chemical analysis.

\section{Numerical calculations DDA method}

DDA is a powerful and flexible method for describing the farfield and near-field properties of targets with arbitrary geometries in a complex dielectric environment [19-21]. In DDA, the continuum target is represented by a finite cubic array of polarizable point dipoles, which is excited by an applied EM field. Each dipole interacts with both of the external field and the induced electric fields generated by all other dipoles in this array. The response of this array to the incident light is then solved self-consistently by using Maxwell's equations. Recently, an extension of DDA to periodic structures has been developed, allowing for the calculation of the optical properties of $1 \mathrm{D}$ and 2D arrays. The theoretical principle of the DDA for periodic targets has been described in more detail elsewhere [22]. Briefly, a "target unit cell" (TUC), repeated in single or double directions, is utilized to assemble the periodic array. In this case, each dipole interacts with the incident electric field and the electric fields scattered by all of the other dipoles in the TUC and the replicas of the TUC. The EM problem is then solved self-consistently through Maxwell's equations. In a recent work, Kim et al. showed that this generalized DDA method was an efficient and versatile numerical approach for calculations of optical properties of AgNR array [23].

To investigate the SERS enhancement of AgNR arrays fabricated by OAD method in terms of EM mechanism, we simulated the local field enhancement of the nanoarrays in vacuum employing the open-source code DDSCAT 7.2 developed by Draine and Flatau [19], which has the capability of performing efficient "near-field" calculations in and around the target by using fast-Fourier transform (FFT) methods [21]. The cubic grid spacing was $3 \mathrm{~nm}$ in all calculations. The dielectric constants of Ag were obtained from the experimental data of Johnson and Christy [24]. The value of the interaction cut-off parameter $\gamma$ was taken to be 0.01 . 


\section{Electromagnetic enhancement factor}

The electromagnetic enhancement factor (EF) is commonly approximated by the following formula [25]:

$$
\mathrm{EF}=\left|\mathbf{E}_{\mathrm{loc}}\left(\mathbf{r}_{\mathrm{m}}, \omega\right)\right|^{2}\left|\mathbf{E}_{\mathrm{loc}}\left(\mathbf{r}_{\mathrm{m}}, \omega^{\prime}\right)\right|^{2},
$$

where $\mathbf{r}_{\mathrm{m}}$ is the location of the molecule, $\mathbf{E}_{\mathrm{loc}}$ is the enhancement of the local electric field (the ratio of the local field to the excitation field associated with the incident plane wave), and $\omega$ and $\omega^{\prime}$ are the incident and Stokes shifted frequencies, respectively. Normally, the shift is small and can be neglected compared to the plasmonic resonance width in metal nanosystems, leading to a fourth-power dependence [26],

$$
\mathrm{EF} \approx\left|\mathbf{E}_{\mathrm{loc}}\left(\mathbf{r}_{\mathrm{m}}, \omega\right)\right|^{4}
$$

To evaluate the EF of SERS for the nanostructures, we calculated the sum and the average of the EF within a unit cell of the periodic lattice over the available surface area except the bottom, which was connected with the supporting substrate, by using $\mathrm{EF}_{\text {sum }}=\int\left|\mathbf{E}_{\text {loc }}\right|^{4} d S$ and $\mathrm{EF}_{\text {avg }}=\int\left|\mathbf{E}_{\text {loc }}\right|^{4} d S / \int d S$, respectively [27]. Note that the value of $\left|\mathbf{E}_{\text {loc }}\right|$ was not calculated exactly at the particle surface, but half a grid point (i.e., $1.5 \mathrm{~nm}$ ) away from each exposed cube surface.

\section{Models}

The models used here are similar to those published previously in [16]. Figure 1 illustrates the regular hexagonal pattern substrate and four different target units considered in the calculations with the parameters shown on the schematic, selected from possible nanorod array structures fabricated by the guided OAD method [16]. The nanorods were arranged in the hexagonal lattice with a centre-to-centre distance of $300 \mathrm{~nm}$ unless otherwise noted (Figure 1a). The orientation of the oblique nanorods was chosen to be along the $y$-direction, and the tilting angle was set to $42^{\circ}$ relative to the $y$-direction [16]. The upper oblique parts of the nanorods were all modelled as tilted cylinders with a hemispherical cap at each end, in order to avoid the "lightening rod effect" at the top edges of the nanorods in the electrodynamics simulations. The gaps between adjacent nanorods along the $y$-direction were fixed to $21 \mathrm{~nm}$ unless specified otherwise, resulting in a cylinder with a diameter of $187 \mathrm{~nm}$. When investigating the effect of different structures on the SERS enhancement, the volume of each target unit was kept constant. This was achieved by considering a factor of $\sin \left(42^{\circ}\right)$ when designing the height of the vertical pillar base in S0:42 and S0:-42:42. The nominal aspect ratio (AR), defined as $l_{1} /(187 \mathrm{~nm})$, was used for all structures. For simplicity, the supporting substrates of the arrays were not considered in the simulations. Only the 2D AgNR array of S42 with AR $=3.5$ and the excitation wavelength of $632.8 \mathrm{~nm}$ were investigated (a)

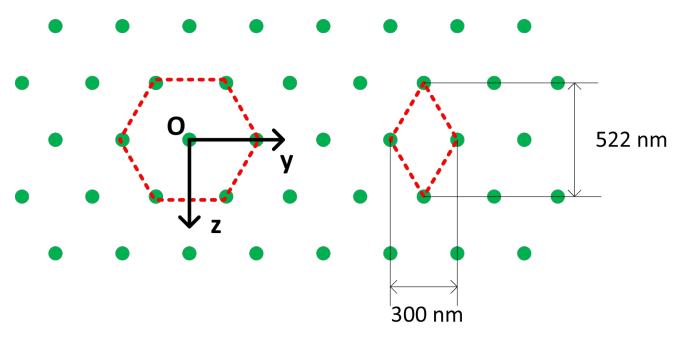

(b)

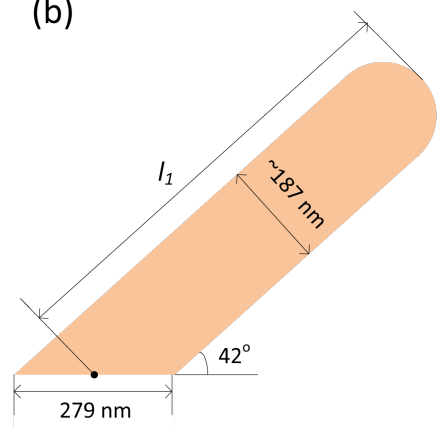

(c)

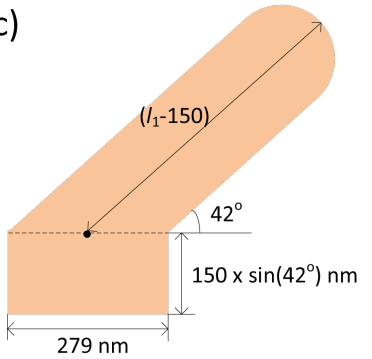

(d)

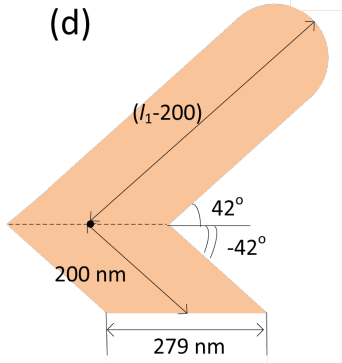

(e)

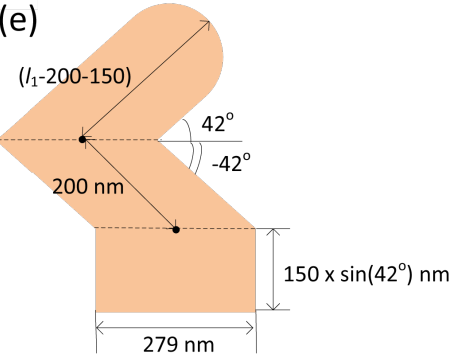

Figure 1: Schematic of the hexagonal pattern substrate (a) and four different target units: (b) S42; (c) S0:42; (d) S-42:42; (e) S0:-42:42. 
except in the sections of structure dependence and excitationwavelength dependence.

\section{Results and Discussion \\ Extinction for isolated nanorods and nanorod arrays}

Typically, metal nanoparticle with anisotropic structure shows multiple plasmon resonances associated with different modes under appropriate excitations [20]. For AgNRs much smaller than the wavelength of light, the extinction spectra usually exhibit a transverse mode centred at around $420 \mathrm{~nm}$ and a longitudinal mode in the range of 500-1100 nm depending on the AR $[28,29]$. These are considered to arise from the dipole plasmon resonances. For AgNRs of large sizes, however, higher order modes of plasmon resonances can be excited [20]. As the target units investigated in the arrays consist of tilted rods, it is expected that both transverse and longitudinal modes can be excited when they are illuminated under normal incidence. Here, the normal incidence is defined as the light with the propagation direction parallel to the surface normal of the substrate (perpendicular to the $y$-direction).

Figure 2 shows typical extinction efficiency spectra of an isolated S42 AgNR of AR 3.5. Under normal incidence of p-polarization, the extinction spectrum has a broad band starting from $320 \mathrm{~nm}$. A general trend of slow increase in the efficiency is apparent in the range of $400-800 \mathrm{~nm}$, with some noticeable features at around 380,440 and $680 \mathrm{~nm}$. In order to identify the

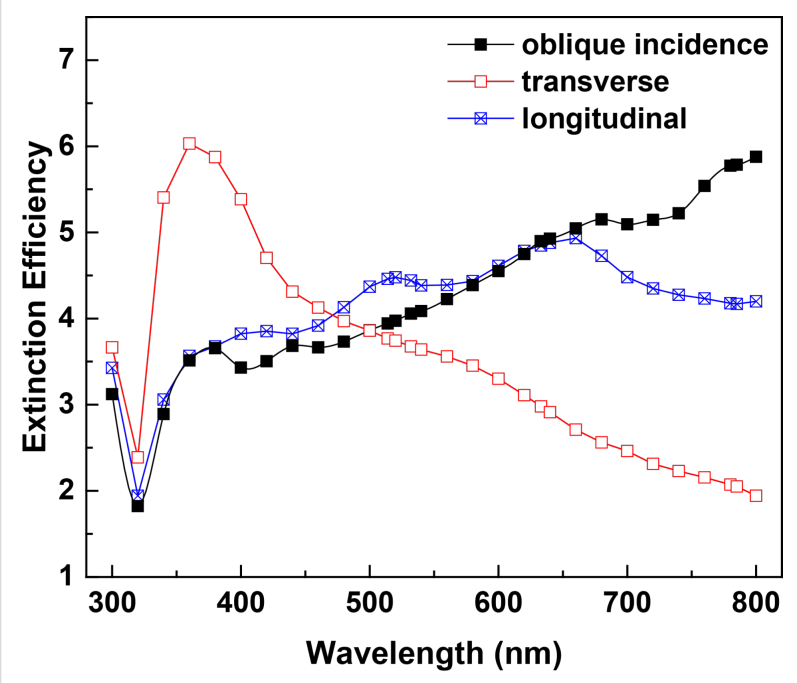

Figure 2: Extinction efficiency spectra of isolated S42 AgNR with $A R=3.5$. The black curve corresponds to the situation in which the AgNR has a tilting angle of $42^{\circ}$ and the propagation direction of the p-polarized light is parallel to the substrate surface normal. The red and blue curves represent the extinction efficiency of the target under the excitation of s-polarization and p-polarization, respectively, when the propagation direction of the light is perpendicular to the long axis of the nanorod. plasmon modes, the extinction efficiency spectra of the target under the s-polarized and the p-polarized excitations are also depicted in Figure 2, in which the propagation direction of the light is perpendicular to the long axis of the nanorod. A major plasmon resonance peak centred at $360 \mathrm{~nm}$ is found under the excitation of s-polarization, along with a broad shoulder at around $550 \mathrm{~nm}$. These resonances can be assigned as dipole $(550 \mathrm{~nm})$ and quadrupole $(360 \mathrm{~nm})$ plasmon modes, respectively, as found in Ag nanoparticles of large sizes [20]. In the case of p-polarized excitation, the extinction spectra has a broad band ranging from 320 to $800 \mathrm{~nm}$, with three distinguishable peaks located at around 400, 520 and $660 \mathrm{~nm}$. Generally, the number of plasmon modes increases with the increasing of asymmetry. The resonance at $660 \mathrm{~nm}$ is ascribed to the dipole plasmon mode, while the resonances at $520 \mathrm{~nm}$ and $400 \mathrm{~nm}$ may be related to higher-order multipolar plasmon modes. Obviously, the extinction efficiency spectra of the tilted target unit under the normal incidence of p-polarization consist of both transverse and longitudinal modes.

As the target units form 2D arrays, the optical properties change due to the coupling effect between neighbouring rods, as depicted in Figure 3. Interestingly, the extinction spectra of the S42 and the S-42:42 arrays are almost the same, so are those of the S0:42 and the S0:-42:42 arrays, although the optical spectra of corresponding individual target units are different from each other. This is probably due to the strong coupling effect resulted from the narrow gap between nanorods investigated here. Significant increases of absorption efficiency are found for all four arrays, (almost doubled in the ranges of 400-700 nm for S42/S-42:42, and 400-640 nm for S0:42/S0:-42:42 arrays), in comparison to that of individual target units. Contributions from absorption and scattering are comparable in these ranges. At long wavelengths, (740 nm for S42/S-42:42; $700 \mathrm{~nm}$ for S0:42/S0:-42:42 arrays), absorption decreases and scattering dominates the extinction spectra. The dependence of the scattering on the wavelength shows clear oscillations, different from that of individual target units. Moreover, the scattering efficiencies are significantly reduced, resulting in much decreased extinction efficiencies in comparison with that of individual targets.

\section{Effects of structure and length}

As shown in the previous section, both transverse and longitudinal modes in the tilted nanorods can be excited simultaneously by the p-polarized light under normal incidence. The coupling of EM fields of neighboring rods greatly enhances the local fields, forming so-called "hotspots". Figure 4 shows the calculated contours of EF for AgNR 2D hexagonal arrays of different structures with $A R=3.5$. Multiple hotspots are found distributed in the gaps. However, the number and the 

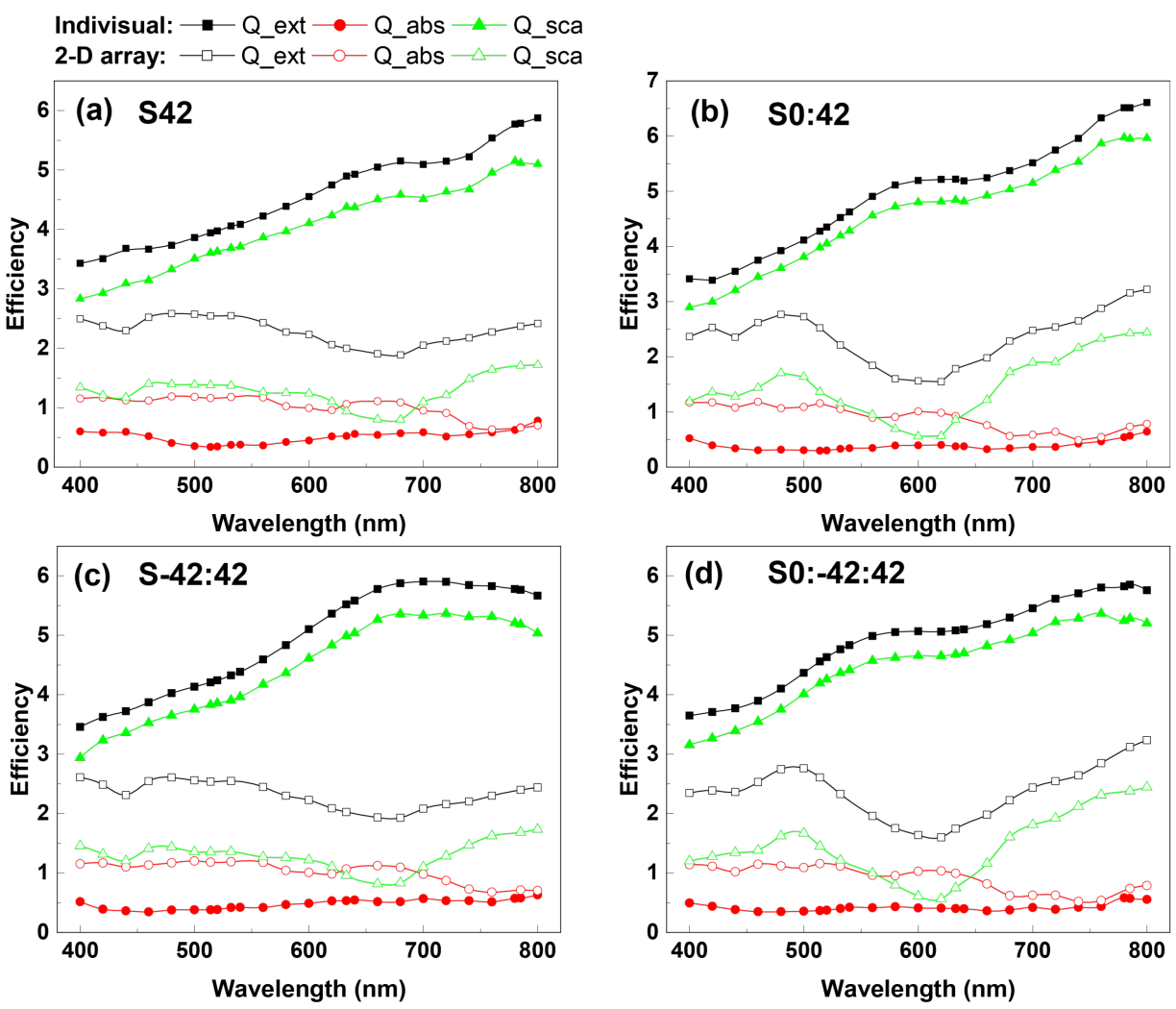

Figure 3: Extinction, absorption and scattering efficiencies of the four target units with $A R=3.5$ and their $2 D$ hexagonal arrays with a periodicity of $300 \mathrm{~nm}$ : (a) S42, (b) S0:42, (c) S-42:42, (d) S0:-42:42. The light is incident along the substrate surface normal with p-polarization.
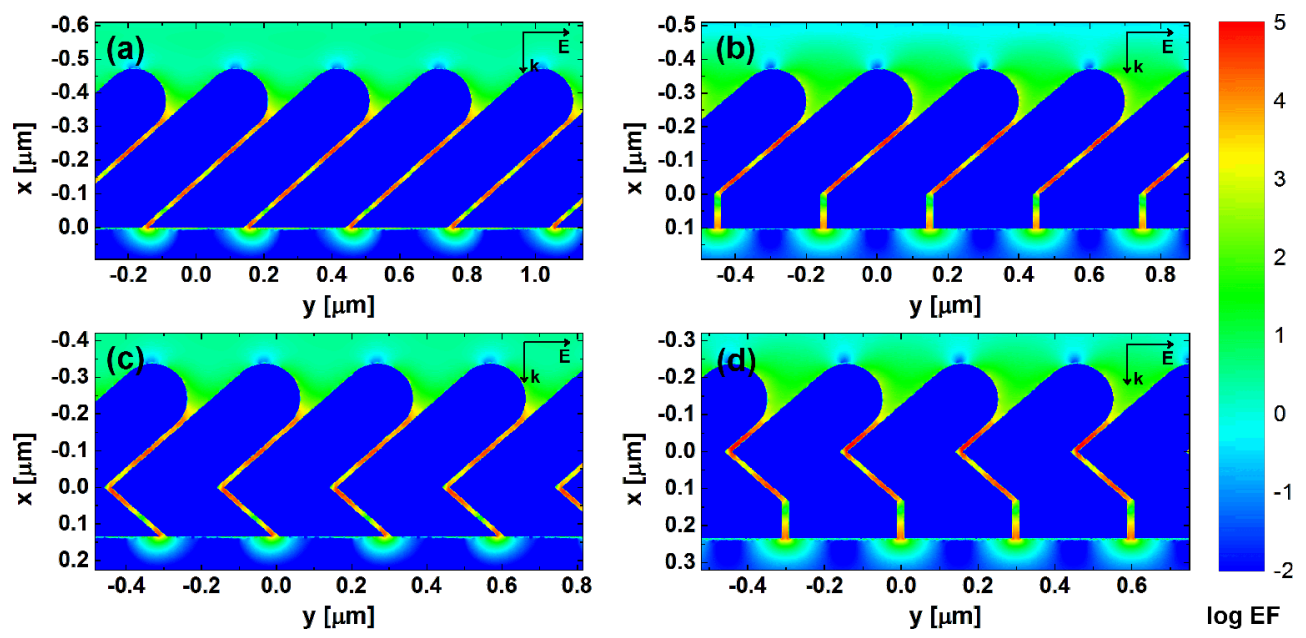

Figure 4: EF distributions obtained from DDA calculations for AgNR 2D hexagonal arrays of different structures with AR = 3.5: (a) S42, (b) S0:42, (c) S-42:42, (d) S0:-42:42. The colour bar is at log scale. The excitation wavelength is $632.8 \mathrm{~nm}$ and the polarization is parallel to the $y$-direction. All internal fields are set to 0.01 for visual clarity.

intensity of the hotspots are structure-dependent. Both S42 and $\mathrm{S}-42: 42$ have four hotspots in each gap, while S0:42 and S0:-42:42 have three hotspots between adjacent nanorods. The brightest hotspots are found in S0:42 and S0:-42:42. Quan- titative analysis shows that the average EFs of S42 and S-42:42 are comparable, which are 797 and 793 , respectively, while S0:-42:42 shows the strongest $\mathrm{EF}_{\mathrm{avg}}$ of 1228 , followed by $\mathrm{S} 0: 42$ with $\mathrm{EF}_{\mathrm{avg}}=1006$. Corners/bends are usually considered 
to give rise intense fields for SERS due to the "lightening rod effect" [5,6,17]. However, similar EFs from S42 and S-42:42, as well as S0:-42:42 and S0:42, show that there are no significant contribution from near-field enhancement right at the corners/bends. This indicates that strong EM coupling in the narrow gap is the dominant factor for the near-field enhancement in these arrays.

We further investigated the dependence of EF on the length of AgNR in different structures. A range of aspect ratios from 2.0 to 5.0 was chosen for S42 arrays, while ARs ranging from 3.0 to 5.0 were applied to other structures due to the constraints of the structure parameters investigated in this work. The number of "hotspots" between adjacent nanorods was found to increase in all four structures as their ARs increased. The $\mathrm{EF}_{\mathrm{avg}}$ and $\mathrm{EF}_{\text {sum }}$ of each structure with varying ARs are shown in Figure 5a and Figure 5b, respectively. It is interesting to find that the EFs of S0:42 and S0:-42:42 exhibit a similar behavior as the AR increases, both have a general decreasing trend but in an oscillating manner. The $\mathrm{EF}_{\mathrm{avg}}$ of $\mathrm{S} 42$ reaches its maximum at $\mathrm{AR}=2.5$, more than four times than that in the case of
$\mathrm{AR}=5.0$. It is worth noting that the EFs of S42 and S-42:42 are comparable at the same AR region between 3.0 and 5.0. And both of their $\mathrm{EF}_{\mathrm{avg}}$ decrease as the AR increases, consistent with the simulation result from $\mathrm{Cu}$ nanorod arrays in our previous work [16]. As the increase of surface area can result in an increased amount of molecular adsorbate and in turn an enhanced SERS intensity, here we take the surface area effect into account and compare the total SERS enhancement $\left(\mathrm{EF}_{\text {sum }}\right)$. As shown in Figure 5b, the surface effect is clearly visible at certain ARs and seems also depending on the structures of target units, although $\mathrm{EF}_{\text {sum }}$ shows a similar trend against $\mathrm{AR}$ as $\mathrm{EF}_{\mathrm{avg}}$ does.

\section{Effect of the excitation wavelength}

Since the SERS effect is a near-field phenomenon and related to the localized surface plasmon resonance (LSPR) of the nanostructures, it is expected to exhibit a behavior that depends on the excitation wavelength. Here, we calculated the EFs of the S42 AgNR arrays with the commonly used excitation wavelengths, i.e., 514, 532, 632.8 and 785 nm, as shown in Figure 6. As can be seen from Figure 6a, the excitation of $532 \mathrm{~nm}$ gives
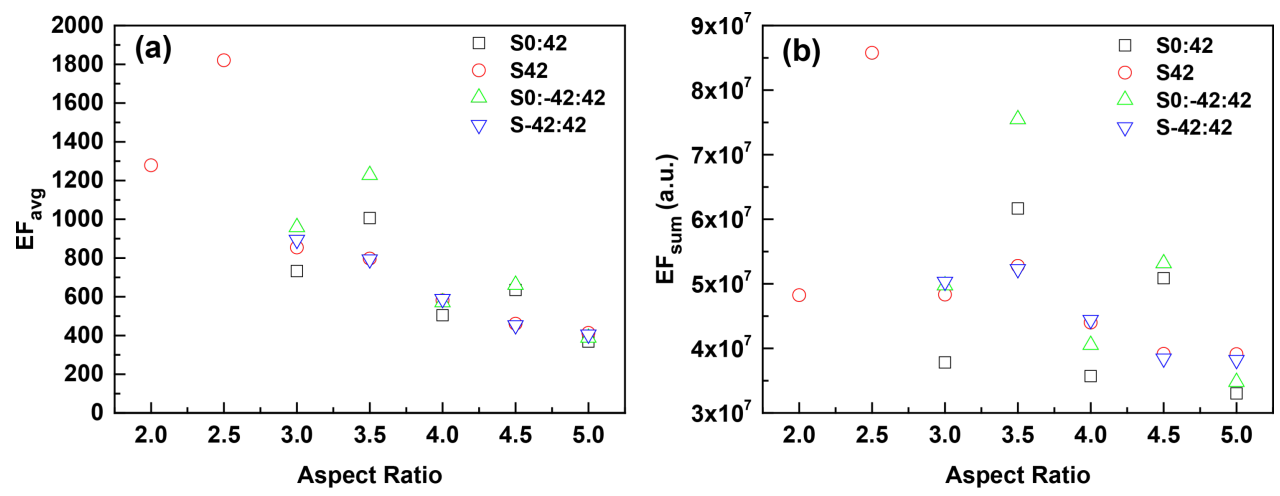

Figure 5: The average EFs (a) and the total EFs (b) of AgNR 2D hexagonal arrays with different structures and ARs. The excitation wavelength is $632.8 \mathrm{~nm}$ and the polarization is parallel to the $y$-direction.
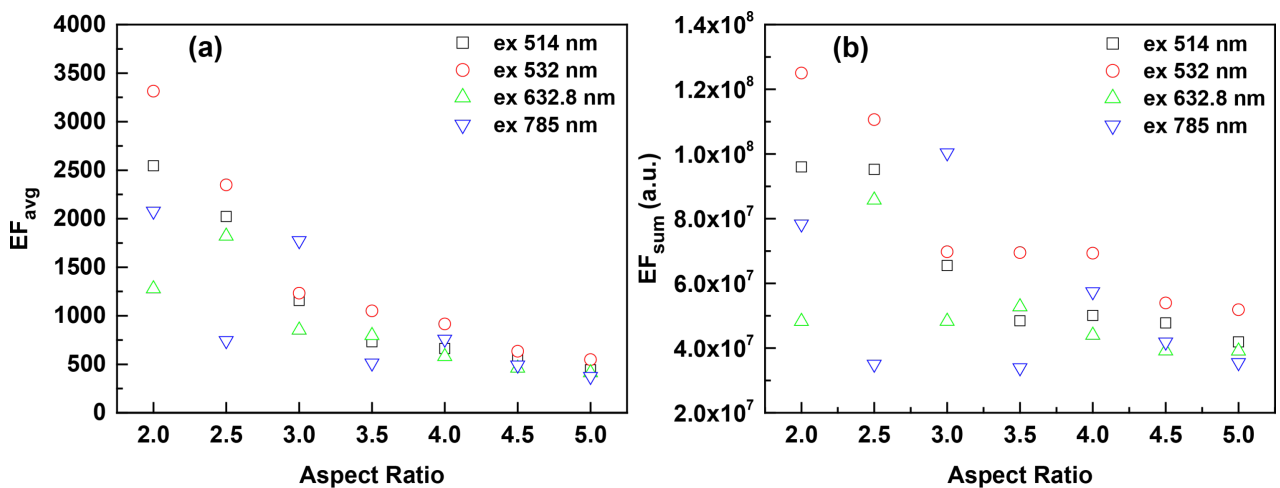

Figure 6: The average EFs (a) and the total EFs (b) of S42 AgNR 2D hexagonal arrays with different ARs, illuminated by different excitation wavelengths (i.e., 514, 532, 632.8 and $785 \mathrm{~nm}$ ) with the polarization parallel to the $y$-direction. 
the most intense $\mathrm{EF}_{\mathrm{avg}}$ at each $\mathrm{AR}$ except $\mathrm{AR}=3.0$. The $\mathrm{EF}_{\mathrm{avg}}$ of the $\mathrm{AR}=2.0$ array illuminated by $532 \mathrm{~nm}$ is more than twice than that of $632.8 \mathrm{~nm}$. It is interesting that the differences of $\mathrm{EF}_{\mathrm{avg}}$ between different excitation wavelengths become insignificant at large ARs. The $\mathrm{EF}_{\mathrm{avg}}$ decreases under both excitations of 514 and $532 \mathrm{~nm}$ as the AR increases, while the $\mathrm{EF}_{\mathrm{avg}}$ shows an oscillating behavior at low ARs in the cases of 632.8 and $785 \mathrm{~nm}$ excitations. Notably, the array with $\mathrm{AR}=2.0$ excited by $532 \mathrm{~nm}$ exhibits the most intense $\mathrm{EF}_{\text {sum }}$ despite of its relative small surface area, as shown in Figure 6 b.

In order to understand the wavelength dependence of the EM enhancement, the extinction and absorption efficiency spectra of S42 AgNR array with varying ARs were also calculated and are given in Figure 7. It is clear that there is no direct correlation between the extinction efficiency and the average EF or the total EF. However, the dependence of absorption efficiency on the AR at each excitation wavelength shows a similar trend as the total EF. Typical features, such as oscillating behavior at low ARs in the cases of 632.8 and $785 \mathrm{~nm}$ excitations and highest efficiency at $\mathrm{AR}=2.0$ under $532 \mathrm{~nm}$ excitation, are consistent with what was observed in Figure 6. It suggests that absorption efficiency could be used as an indicator for SERS enhancement. Nevertheless, the connection between the absorption/extinction spectra and the enhancement in SERS is still not fully understood [30] and requires further investigation.

\section{Effect of incident angle}

As is evident in Figure 8, the $\mathrm{EF}_{\text {avg }}$ strongly depends on the incident angle. The incident angle is defined as the angle with respect to the surface normal, as illustrated in the insert of Figure 8. The most intense $\mathrm{EF}_{\mathrm{avg}}$ is obtained when the array is illuminated at a positive angle of about $10^{\circ}\left(38^{\circ}\right.$ towards the long axis of nanorod). At this angle, the incident direction is

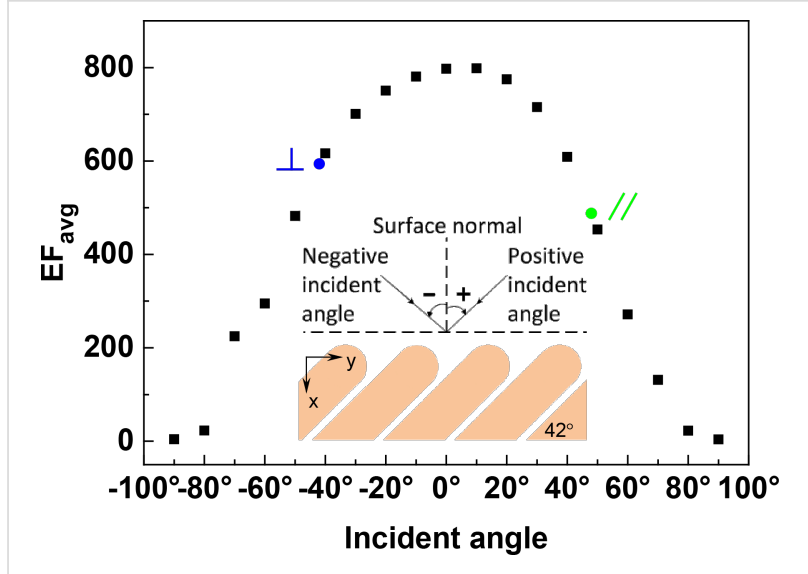

Figure 8: The angular dependent $\mathrm{EF}_{\text {avg }}$ of $\mathrm{S} 42 \mathrm{AgNR} 2 \mathrm{D}$ hexagonal array with $A R=3.5$. The excitation wavelength is $632.8 \mathrm{~nm}$. The insert illustrates the incident direction and angle. The incident polarization is in the $x-y$ plane and perpendicular to the propagation. The symbols, $\perp$ and $/ /$, denote that the incident directions are perpendicular and parallel to the long axis of the nanorods, respectively.

neither parallel nor perpendicular to the long axis of the nanorods. The $\mathrm{EF}_{\mathrm{avg}}$ decreases dramatically when the incident angle deviates from the optimum value. A similar asymmetric angular dependence of the SERS response was experimentally observed by Liu et al. in a tilted AgNR array with a tilting angle of ca. $17^{\circ}$ [31], for which the maximum SERS intensity was obtained at an incident angle of about $45^{\circ}$ off the surface normal. A modified Greenler's model was also proposed to interpret this phenomenon. In this model, the molecule adsorbed on the side of the nanorod is treated as a dipole perpendicular to the long axis of the nanorod, while the surface of the nanorod was considered as a planar surface. The SERS intensity was assumed to be proportional to the mean square of total scattered field that was calculated by using classical electrodynamics. According to this model, the optimal incident angle
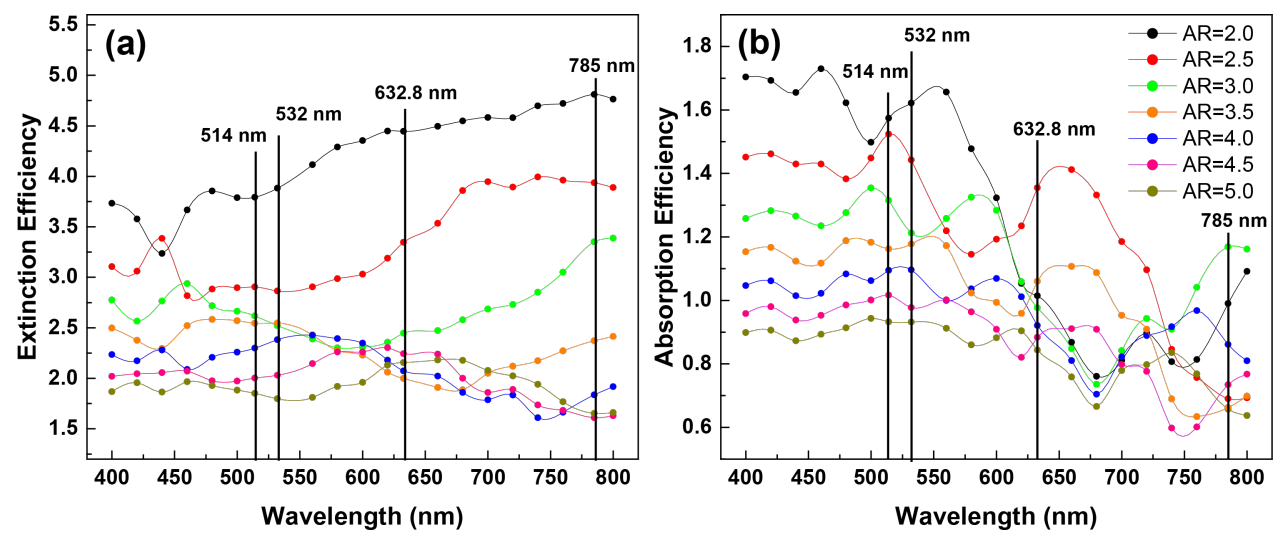

Figure 7: (a) Extinction efficiency spectra of S42 AgNR 2D hexagonal array with AR ranging from 2.0 to 5.0; (b) absorption efficiency spectra of S42 AgNR array with AR ranging from 2.0 to 5.0. The incident polarization is parallel to the $y$-direction. Vertical solid lines indicate the excitation laser wavelengths used in Figure 6. 
increases as the tilting angle of nanorod (with respect to the surface normal) decreases [32]. Therefore, it is not surprising that the optimal incident angle found in our simulation is smaller than that reported in [31]. In fact, the angular dependence of near-field enhancement was also found in the vertical AgNR arrays. It has been revealed that different modes of surface plasmon resonance can only be excited by certain angles of incidence, leading to different near-field enhancements [23,33].

\section{Effect of incident polarization}

Figure 9a shows the polarization dependence of $\mathrm{EF}_{\text {avg }}$ from $\mathrm{S} 42$ AgNR hexagonal array with $\mathrm{AR}=3.5$. The excitation wavelength is $632.8 \mathrm{~nm}$, and the wave vector is perpendicular to the substrate. The polarization angle is defined as the angle between the electric-field vector and the $y$-axis as shown in Figure 1a. The most intense $\mathrm{EF}_{\mathrm{avg}}, 797$, occurs at polarization angles of 0 and $180^{\circ}$. This is caused by the strongest EM coupling effect between adjacent nanorods when the exciting electric field vector is polarized along the interparticle axis ( $y$-axis), as is well known in the particle dimer system [34,35]. The $\mathrm{EF}_{\mathrm{avg}}$ of the array is quite sensitive to the polarization. As the polarization deviates from 0 and $180^{\circ}$, the $\mathrm{EF}_{\text {avg }}$ rapidly decreases, reaching a minimum value of 44 at polarization angles of 90 and $270^{\circ}$.

The polarization dependence of the optical cross sections corresponding to Figure 9a is shown in Figure 9b. The efficiency factors of absorption, scattering and extinction are defined as the ratios of the total cross sections for absorption, scattering and extinction per TUC to the geometrical crosssection of equal-volume sphere in one TUC, respectively [22]. It is found that the absorption shows a polarization with maxima at 0 and $180^{\circ}$, opposite to scattering and extinction that reach the maxima at polarization angles of 90 and $270^{\circ}$ and the minima at 0 and $180^{\circ}$. Interestingly, the absorption follows the same polarization dependence as the $\mathrm{EF}_{\mathrm{avg}}$, while the scattering and extinction exhibit a different behavior. Previously, Zhao et al. observed that the anisotropy of the SERS polarization was different from that of the polarized UV-vis absorbance of a nonplanar AgNR array substrate [36]. Practically, the UV-vis absorption spectrum measured in the experiment is the sum of absorption and scattering, i.e., extinction. So, the experimental observation is in line with this simulation result. The simulation result also suggests that the absorption rather than the extinction or scattering could be an indicator of EM enhancement in SERS performance, in line with the observation in Section "Effect of excitation wavelength".

\section{Effect of lateral gap size}

$\mathrm{EF}_{\mathrm{avg}}$ is highly sensitive to gap size, especially to small gap sizes below $15 \mathrm{~nm}$, as shown in Figure 10. There is a dramatic decrease of $\mathrm{EF}_{\mathrm{avg}}$ with the increase of gap size from 9 to $18 \mathrm{~nm}$, and a much slower decrease with further increase of gap size.

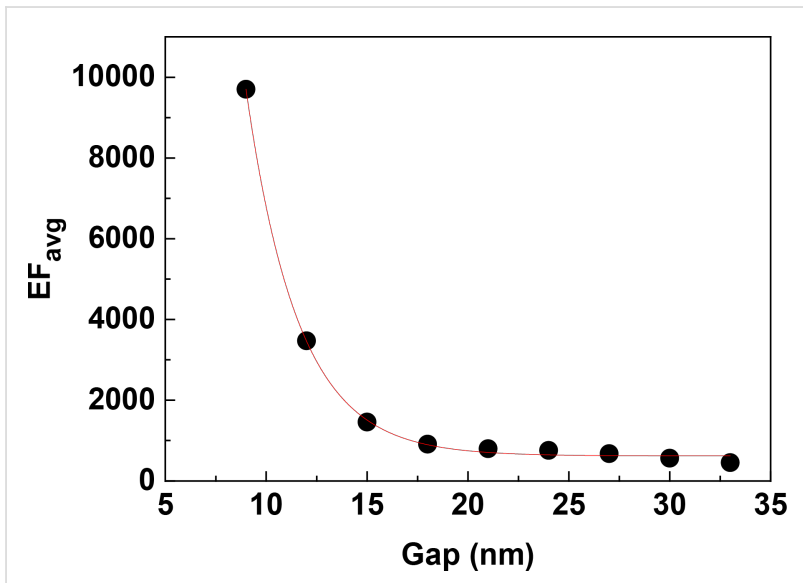

Figure 10: The dependence of $\mathrm{EF}_{\mathrm{avg}}$ on the gap size along the $y$-direction in S42 AgNR 2D hexagonal array with $A R=3.5$. The solid curve is an exponential fitting result. The excitation wavelength is $632.8 \mathrm{~nm}$ and the polarization is parallel to the $y$-direction. (a)

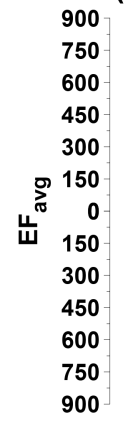

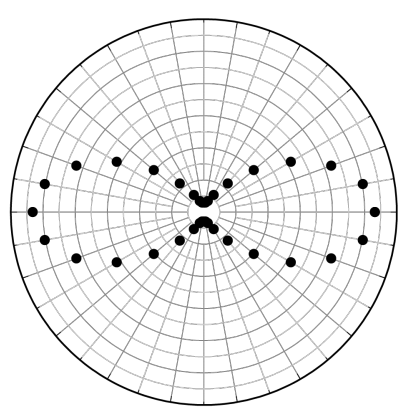

(b)

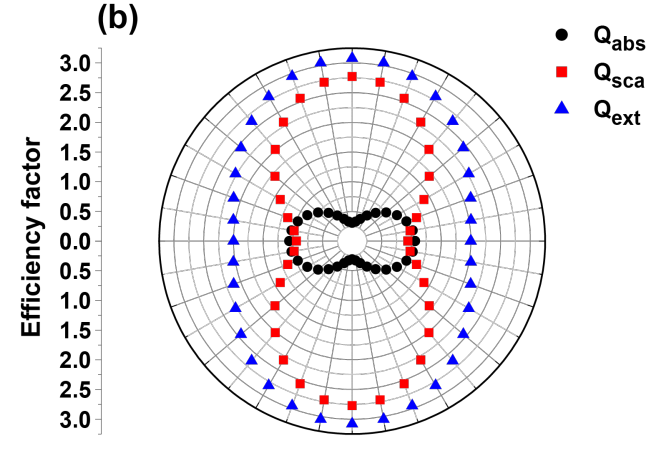

Figure 9: The polarization-dependent $\mathrm{EF}_{\mathrm{avg}}$ (a) and the corresponding absorption (black), scattering (red) and extinction (blue) efficiency factors (b) of S42 AgNR 2D hexagonal array with $A R=3.5$. The excitation wavelength is $632.8 \mathrm{~nm}$. 
The gap size has been a crucial parameter of the SERS substrates because of the strong EM coupling effect at the nanometre scale [37-39]. Due to the challenges of fabricating ordered AgNR arrays by the OAD method, the effect of gap size in those arrays have not been experimentally investigated, yet. However, semi-ordered AgNR arrays were obtained by an OAD technique employing 2D Au nano-post arrays in square lattice as seed patterns [40]. The SERS intensities were shown to increase monotonically with the decreasing separation of AgNRs [40], which is consistent with our simulation results.

\section{Random vs ordered arrays}

Although the tilted AgNR arrays fabricated by the OAD method were shown to have SERS enhancement factors greater than $10^{8}$, they were randomly distributed $[12,13]$, which presents a challenge towards highly uniformed and reproducible SERS substrates. Hence, efforts have been devoted to produce wellpatterned AgNR arrays $[16,40]$. It is interesting to compare the EFs of random and ordered arrays through theoretical simulations. However, due to the complexity of the $2 \mathrm{D}$ arrays, it is difficult to model a truly random AgNR array. Here, target units consisting of six AgNRs arranged in the $y$-direction with different gap sizes are used to model the $2 \mathrm{D}$ random arrays. The averages of the gap sizes in the target units are $21 \mathrm{~nm}$, and the gap sizes between the target units along the $y$-direction are set to $21 \mathrm{~nm}$, so that the average gap size along the $y$-direction is the same as that of the 2D ordered array. The gap sizes and the standard deviations (STDEVs) are shown in Table 1.

\begin{tabular}{|c|c|c|c|c|c|c|}
\hline case & $\begin{array}{l}\text { gap } 1 \\
(\mathrm{~nm})\end{array}$ & $\begin{array}{c}\text { gap } 2 \\
(\mathrm{~nm})\end{array}$ & $\begin{array}{c}\text { gap } 3 \\
(\mathrm{~nm})\end{array}$ & $\begin{array}{c}\text { gap } 4 \\
(\mathrm{~nm})\end{array}$ & $\begin{array}{c}\text { gap } 5 \\
(\mathrm{~nm})\end{array}$ & $\begin{array}{l}\text { STDE } \\
\mathrm{V}(\mathrm{nm})\end{array}$ \\
\hline 1 & 21 & 24 & 18 & 27 & 15 & 4.7 \\
\hline 2 & 21 & 30 & 12 & 24 & 18 & 6.7 \\
\hline 3 & 21 & 30 & 12 & 27 & 15 & 7.6 \\
\hline 4 & 21 & 24 & 18 & 33 & 9 & 8.7 \\
\hline 5 & 21 & 27 & 15 & 33 & 9 & 9.5 \\
\hline 6 & 21 & 30 & 12 & 33 & 9 & 10.6 \\
\hline
\end{tabular}

As shown in Figure 11, it is interesting that the EFs of the random arrays are all higher than that of the ordered array. Moreover, the EF increases monotonically as the STDEV of the gap size increases. Remarkably, the random array with a gap size STDEV of $10.6 \mathrm{~nm}$ shows a more than three times stronger $\mathrm{EF}$ than that of the ordered one. This indicates that the random arrays with the same average gap size $(21 \mathrm{~nm})$ as the ordered one can show a better SERS performance, which is consistent with the exponential dependence of the EF on the gap size, as demonstrated in Figure 10. This again manifests the signifi- cance of hotspots in defining total SERS intensity as revealed by Fang et al. experimentally [6]. It is worth pointing out that the difference of EF between random and ordered array is less significant when the average gap size is large and STDEV is small, because the gap sizes are then out of the region of rapidly changing EFs (Figure 10).

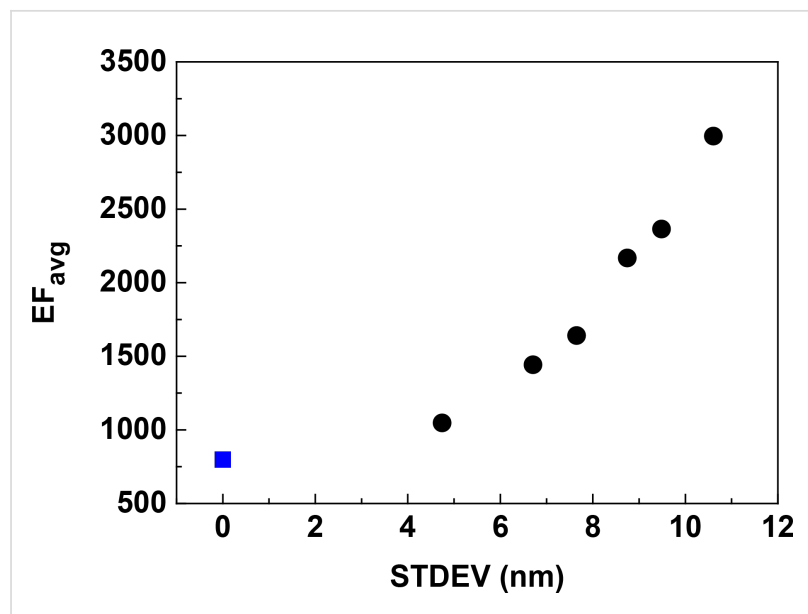

Figure 11: The dependence of $\mathrm{EF}_{\mathrm{avg}}$ on the standard deviation of the gap size along the $y$-direction in the S42 AgNR 2D random array with $A R=3.5$. The average gap size is $21 \mathrm{~nm}$ in the $y$-direction. The excitation wavelength is $632.8 \mathrm{~nm}$ and the polarization is parallel to the $y$-direction. The blue square in the figure indicates the ordered one with the gap size of $21 \mathrm{~nm}$ in the $y$-direction.

\section{Effect of diagonal periodicity}

We have shown that the variations of the gap size in the $y$-direction have a strong influence on the EF of SERS. However, the EF of a 2D array depends not only on the periodicity in the $y$-direction (denoted as lateral periodicity) but also on the periodicity in the diagonal directions. Here, we fixed the lateral periodicity to $300 \mathrm{~nm}$, and investigated the dependence of EF on the diagonal periodicity. Figure 12 shows that a smaller diagonal periodicity, i.e., smaller gap size, does not necessarily result in stronger EF in the $2 \mathrm{D}$ arrays. In fact, the $\mathrm{EF}$ of the $2 \mathrm{D}$ array oscillates as the diagonal periodicity increases from 234 to $1239 \mathrm{~nm}$ (diagonal gap size varying from 21 to $1050 \mathrm{~nm}$ ). The $\mathrm{EF}_{\mathrm{avg}}$ of the ordered array arranged in a regular hexagonal pattern is more than three times lower than that of the ordered array with the diagonal periodicity of $463 \mathrm{~nm}$. It is clear that diagonal periodicity plays an important role in the SERS enhancement for the $2 \mathrm{D}$ array but the dependence of $\mathrm{EF}$ on the diagonal gap is more complicated than that on the lateral gap and the mechanism needs further investigations. Nevertheless, this simulation indicates a new dimension to design OAD AgNR arrays for optimized SERS performance.

Also shown in Figure 12 are the absorption, scattering and extinction efficiency factors at the excitation wavelength of 


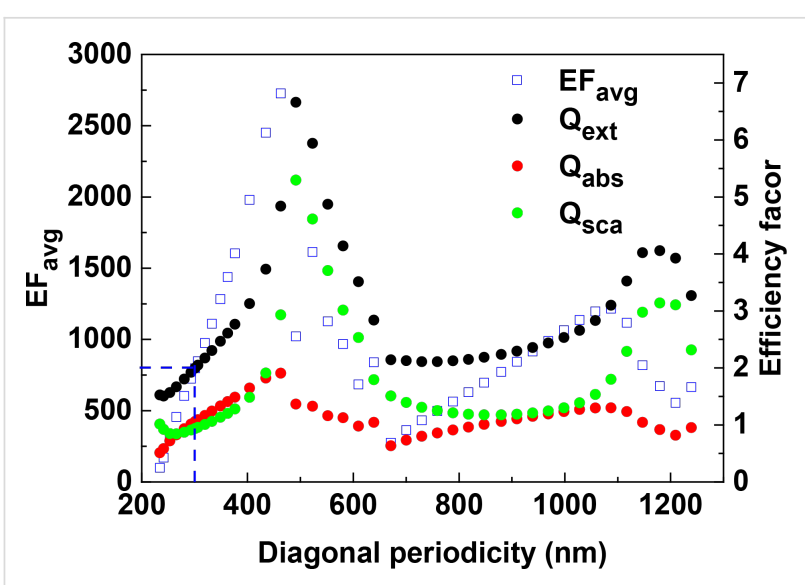

Figure 12: The dependence of $\mathrm{EF}_{\mathrm{avg}}$ and extinction, absorption and scattering efficiency factors on the diagonal periodicity in the $\$ 42$ AgNR 2D array with $A R=3.5$. The excitation wavelength is $632.8 \mathrm{~nm}$ and the polarization is parallel to the $y$-direction. The dashed lines indicate the diagonal periodicity of the ordered array arranged in a regular hexagonal pattern.

$632.8 \mathrm{~nm}$. It clearly demonstrates that absorption follows a similar trend as the $\mathrm{EF}_{\mathrm{avg}}$ does, different from extinction or scattering. This becomes more obvious for a diagonal periodicity larger than $600 \mathrm{~nm}$. Recently, Near et al. found that the field strength within a plasmon mode trends with the absorption in a silver nanocube [41], which is in line with this simulation. This result is also consistent with our simulations on incident polarization effect (Figure 9), indicating that the absorption rather than the extinction or scattering may be a good indicator of the EM enhancement.

\section{Conclusion}

The enhancement factor of SERS of 2D hexagonal silver nanorod arrays was investigated by using the discrete dipole approximation method. The computational studies clearly showed that "hotspots" were distributed in the gaps between adjacent nanorods, and the narrow gaps resulted in strong EFs. The excitation of $532 \mathrm{~nm}$ gives the most intense $\mathrm{EF}_{\mathrm{avg}}$ at each $\mathrm{AR}$ except $\mathrm{AR}=3.0$, and the array with $\mathrm{AR}=2.0$ excited by $532 \mathrm{~nm}$ showed the most intense $\mathrm{EF}_{\text {sum }}$ despite of the smallest surface area. However, the influence of different excitation wavelengths on the EF became insignificant as the AR was over 4.0. The EF was found to be strongly dependent on the polarization of the incident light. The most intense EF was obtained when the array is illuminated with an incident angle about $10^{\circ}$ off the surface normal. The simulations of AgNR arrays of different lengths revealed that increasing rod length generated more "hotspots" but not necessarily increased EF. The EM enhancement of 2D random AgNR arrays was compared with that of an ordered array of the same average gap size. It was found that the average EF of random arrays was stronger than that of an ordered one with the same average gap of $21 \mathrm{~nm}$, which can be explained by the exponential dependence of the average EF on the lateral gap size. Although the narrow lateral gap results in strong EF, the dependence of EF on the diagonal gap shows an oscillating behavior, which implied that the SERS substrates could be optimized by adjusting the diagonal/longitudinal periodicity. The simulation results also indicated that absorption rather than extinction or scattering could be a good indicator of EM enhancement.

\section{Acknowledgements}

G. W. acknowledges financial support from the China Scholarship Council (CSC). Simulation results were obtained by using the EPSRC funded ARCHIE-WeSt High Performance Computer (http://www.archie-west.ac.uk). EPSRC grant no. $\mathrm{EP} / \mathrm{K} 000586 / 1$.

\section{References}

1. Nie, S.; Emory, S. R. Science 1997, 275, 1102-1106. doi:10.1126/science.275.5303.1102

2. Kneipp, K.; Wang, Y.; Kneipp, H.; Perelman, L. T.; Itzkan, I.; Dasari, R. R.; Feld, M. S. Phys. Rev. Lett. 1997, 78, 1667-1670. doi:10.1103/PhysRevLett.78.1667

3. Camden, J. P.; Dieringer, J. A.; Zhao, J.; Van Duyne, R. P. Acc. Chem. Res. 2008, 41, 1653-1661. doi:10.1021/ar800041s

4. Moskovits, M. Rev. Mod. Phys. 1985, 57, 783-826. doi:10.1103/RevModPhys.57.783

5. Moskovits, M. J. Raman Spectrosc. 2005, 36, 485-496. doi:10.1002/jrs.1362

6. Kleinman, S. L.; Frontiera, R. R.; Henry, A.-I.; Dieringer, J. A.; Van Duyne, R. P. Phys. Chem. Chem. Phys. 2013, 15, 21-36. doi:10.1039/c2cp42598j

7. Fang, Y.; Seong, N.-H.; Dlott, D. D. Science 2008, 321, 388-392. doi:10.1126/science.1159499

8. Cang, H.; Labno, A.; Lu, C.; Yin, X.; Liu, M.; Gladden, C.; Liu, Y.; Zhang, X. Nature 2011, 469, 385-388. doi:10.1038/nature09698

9. Banholzer, M. J.; Millstone, J. E.; Qin, L.; Mirkin, C. A. Chem. Soc. Rev. 2008, 37, 885-897. doi:10.1039/b710915f

10. Wang, Y.; Schlücker, S. Analyst 2013, 138, 2224-2238. doi:10.1039/c3an36866a

11. Sharma, B.; Fernanda Cardinal, M.; Kleinman, S. L.; Greeneltch, N. G.; Frontiera, R. R.; Blaber, M. G.; Schatz, G. C.; Van Duyne, R. P. MRS Bull. 2013, 38, 615-624. doi:10.1557/mrs.2013.161

12. Chaney, S. B.; Shanmukh, S.; Dluhy, R. A.; Zhao, Y.-P. Appl. Phys. Lett. 2005, 87, 031908. doi:10.1063/1.1988980

13. Driskell, J. D.; Shanmukh, S.; Liu, Y.; Chaney, S. B.; Tang, X.-J.; Zhao, Y.-P.; Dluhy, R. A. J. Phys. Chem. C 2008, 112, 895-901. doi:10.1021/jp075288u

14. Liu, Y.-J.; Chu, H. Y.; Zhao, Y.-P. J. Phys. Chem. C 2010, 114, 8176-8183. doi:10.1021/jp1001644

15. Song, S.; Keating, M.; Chen, Y.; Placido, F. Meas. Sci. Technol. 2012, 23, 084007. doi:10.1088/0957-0233/23/8/084007

16. Keating, M.; Song, S.; Wei, G.; Graham, D.; Chen, Y.; Placido, F. J. Phys. Chem. C 2014, 118, 4878-4884. doi:10.1021/jp410116h

17. Zhou, Q.; Zhang, X.; Huang, Y.; Li, Z.; Zhao, Y.; Zhang, Z. Appl. Phys. Lett. 2012, 100, 113101. doi:10.1063/1.3694056 18. Liu, Y.-J.; Zhang, Z.-Y.; Zhao, Q.; Dluhy, R. A.; Zhao, Y.-P. J. Phys. Chem. C 2009, 113, 9664-9669. doi:10.1021/jp902142y 
19. Draine, B. T.; Flatau, P. J. J. Opt. Soc. Am. A 1994, 11, 1491-1499. doi:10.1364/JOSAA.11.001491

20. Kelly, K. L.; Coronado, E.; Zhao, L. L.; Schatz, G. C. J. Phys. Chem. B 2003, 107, 668-677. doi:10.1021/jp026731y

21. Flatau, P. J.; Draine, B. T. Opt. Express 2012, 20, 1247-1252. doi:10.1364/OE.20.001247

22. Draine, B. T.; Flatau, P. J. J. Opt. Soc. Am. A 2008, 25, 2693-2703. doi:10.1364/JOSAA.25.002693

23. Kim, S.; Jung, Y.; Gu, G. H.; Suh, J. S.; Park, S. M.; Ryu, S. J. Phys. Chem. C 2009, 113, 16321-16328. doi:10.1021/jp811516s

24. Johnson, P. B.; Christy, R. W. Phys. Rev. B 1972, 6, 4370-4379. doi:10.1103/PhysRevB.6.4370

25. Kerker, M.; Wang, D.-S.; Chew, H. Appl. Opt. 1980, 19, 4159-4174. doi:10.1364/AO.19.004159

26. Stockman, M. I. Electromagnetic Theory of SERS. In Surface-Enhanced Raman Scattering - Physics and Applications; Kneipp, K.; Moskovits, M.; Kneipp, H., Eds.; Topics in Applied Physics, Vol. 103; Springer: Berlin, Germany, 2006; pp 47-65. doi:10.1007/3-540-33567-6_3

27. Li, S.; Pedano, M. L.; Chang, S.-H.; Mirkin, C. A.; Schatz, G. C. Nano Lett. 2010, 10, 1722-1727. doi:10.1021/nl100099g

28. Orendorff, C. J.; Gearheart, L.; Jana, N. R.; Murphy, C. J. Phys. Chem. Chem. Phys. 2006, 8, 165-170. doi:10.1039/b512573a

29. Mahmoud, M. A.; El-Sayed, M. A. J. Phys. Chem. Lett. 2013, 4, 1541-1545. doi:10.1021/jz4005015

30. Le Ru, E. C.; Galloway, C.; Etchegoin, P. G. Phys. Chem. Chem. Phys. 2006, 8, 3083-3087. doi:10.1039/b605292d

31. Liu, Y.; Fan, J.; Zhao, Y.-P.; Shanmukh, S.; Dluhy, R. A. Appl. Phys. Lett. 2006, 89, 173134. doi:10.1063/1.2369644

32. Liu, Y.-J.; Zhao, Y.-P. Phys. Rev. B 2008, 78, 075436. doi:10.1103/PhysRevB.78.075436

33. Evans, P. R.; Kullock, R.; Hendren, W. R.; Atkinson, R.; Pollard, R. J.; Eng, L. M. Adv. Funct. Mater. 2008, 18, 1075-1079. doi:10.1002/adfm.200701289

34. Camden, J. P.; Dieringer, J. A.; Wang, Y.; Masiello, D. J.; Marks, L. D.; Schatz, G. C.; Van Duyne, R. P. J. Am. Chem. Soc. 2008, 130 , 12616-12617. doi:10.1021/ja8051427

35. Shegai, T.; Li, Z.; Dadosh, T.; Zhang, Z.; Xu, H.; Haran, G. Proc. Natl. Acad. Sci. U. S. A. 2008, 105, 16448-16453. doi:10.1073/pnas.0808365105

36. Zhao, Y.-P.; Chaney, S. B.; Shanmukh, S.; Dluhy, R. A. J. Phys. Chem. B 2006, 110, 3153-3157. doi:10.1021/jp057406o

37. Xu, H.; Bjerneld, E. J.; Käll, M.; Börjesson, L. Phys. Rev. Lett. 1999, 83, 4357-4360. doi:10.1103/PhysRevLett.83.4357

38. Zou, S.; Schatz, G. C. Chem. Phys. Lett. 2005, 403, 62-67. doi:10.1016/j.cplett.2004.12.107

39. Wustholz, K. L.; Henry, A.-I.; McMahon, J. M.; Freeman, R. G.; Valley, N.; Piotti, M. E.; Natan, M. J.; Schatz, G. C.; Van Duyne, R. P. J. Am. Chem. Soc. 2010, 132, 10903-10910. doi:10.1021/ja104174m

40. Liu, Y.-J.; Zhang, Z.-Y.; Dluhy, R. A.; Zhao, Y.-P. J. Raman Spectrosc. 2010, 41, 1112-1118. doi:10.1002/jrs.2567

41. Near, R.; Hayden, S.; El-Sayed, M. J. Phys. Chem. C 2012, 116, 23019-23026. doi:10.1021/jp309272b

\section{License and Terms}

This is an Open Access article under the terms of the Creative Commons Attribution License

(http://creativecommons.org/licenses/by/2.0), which permits unrestricted use, distribution, and reproduction in any medium, provided the original work is properly cited.

The license is subject to the Beilstein Journal of Nanotechnology terms and conditions:

(http://www.beilstein-journals.org/bjnano)

The definitive version of this article is the electronic one which can be found at:

doi:10.3762/bjnano.6.69 